\section{Exploring avidity:} understanding the potential gains in functional affinity and target residence time of bivalent and heterobivalent ligands

\author{
Georges Vauquelin ${ }^{1}$ and Steven J Charlton ${ }^{2}$ \\ ${ }^{1}$ Department Molecular and Biochemical Pharmacology, Vrije Universiteit Brussel, Brussels, \\ Belgium, and ${ }^{2}$ Novartis Institutes for Biomedical Research, Horsham, UK
}

\section{Correspondence}

Professor Georges Vauquelin, Molecular and Biochemical Pharmacology Department, Free University of Brussels (VUB), Building E.5.24, Pleinlaan 2, B-1050 Brussel, Belgium. E-mail: gvauquel@vub.ac.be

\section{Keywords}

drug design; pharmacodynamics; bivalent ligand; avidity; residence time; binding mechanism; simulations; rebinding

\section{Received}

2 July 2012

Revised

5 November 2012

Accepted

28 November 2012

Bivalent ligands are increasingly important therapeutic agents. Although the naturally occurring antibodies are predominant, it is becoming more common to combine different antibody fragments or even low molecular weight compounds to generate heterobivalent ligands. Such ligands exhibit markedly increased affinity (i.e. avidity) and target residence time when both pharmacophores can bind simultaneously to their target sites. This is because binding of one pharmacophore forces the second tethered one to stay close to its corresponding site. This 'forced proximity' favours its binding and rebinding (once dissociated) to that site. However, rebinding will also take place when the diffusion of freshly dissociated ligands is merely slowed down. The present differential equation-based simulations explore the way both situations affect ligand binding. Both delay the attainment of binding equilibrium (resulting in steep saturation curves) and also increase the target residence time. Competitive ligands are able to interfere in a concentration-dependent manner, although much higher concentrations are required in the 'forced proximity' situation. Also, it is only in that situation that the ligand shows increased affinity. These simulations shed light on two practical consequences. Depending on the pharmacokinetic half-life of the bivalent ligand in the body, it may not have sufficient time to achieve equilibrium with the target. This will result in lower potency than expected, although it would have significant advantages in terms of residence time. In in vitro experiments, the manifestation of steep saturation curves and of accelerated dissociation in the presence of competitive ligands could mistakenly be interpreted as evidence for non-competitive, allosteric interactions.

\title{
Abbreviations
}

2D, 3D, two- and three-dimensional; (a and c), aa, ab, monovalent, homobivalent and heterobivalent ligands; A, B, target sites (ligand-bound species are named in Schemes 1 and 2); [L], $f$, local concentration of unbound pharmacophore of $\mathrm{ab}$, handicap factor for its binding; $r$, maximal distance between $\mathrm{a}$ and $\mathrm{b}$ in $\mathrm{ab}$

\section{Introduction}

Affinity is the term used to describe the strength of a single bimolecular interaction between a ligand and its target. If a ligand is able to bind to the target via two (or more) pharmacophores, these multiple interactions can synergize to enhance the apparent affinity. This effect is commonly referred to as 'avidity'. This can obviously only take place when the target sites are sufficiently close together for them to be simultaneously occupied by the two pharmacophores of the ligand. The resulting synergy could be brought about via allosteric interactions (Valant et al., 2012), but this is not a necessary condition. Indeed, binding of one pharmacophore to its corresponding site at the target brings the second pharmacophore in close proximity to that target, increasing its local concentration and thereby the probability of an inter- 
action with the second site (Kaufman and Jain, 1992; Plückthun and Pack, 1997; Kramer and Karpen, 1998). This can also lead to large increases in target residence time, because when one pharmacophore dissociates from its target site, it has to remain in 'forced proximity' as long as its tethered, companion pharmacophore is still bound. The resulting high local concentration of the dissociated pharmacophore will therefore also significantly increase its chances to bind again to its original target site. As further expanded on below, this represents a form of 'rebinding'.

The most common examples of bivalent ligands are the natural antibody proteins, IgE, IgG and IgD. These immunoglobulins possess two identical epitope-binding regions and so are considered to be bivalent. Because bivalent ligands have two identical pharmacophores, in order for them to exhibit avidity, they must be able to cross-link the same site on two different targets that are in close proximity to each other. This is in contrast to heterobivalent ligands that possess two different pharmacophores. While still retaining their ability to cross-link two targets, these molecules can also obtain an avidity-driven increase in affinity by binding at two different sites on a single target.

It is becoming more and more common to mimic the multivalency of naturally occurring immunoglobulins when generating new ligands of therapeutic potential, particularly in the combination of ligand fragments. Although the Fc domain on naturally occurring antibodies is critical for the recruitment of cytotoxic effector functions, when it is present on a therapeutic protein, it can sometimes cause inappropriate activation of Fc-receptor expressing cells, leading to toxic levels of cytokine release. To overcome this (and other) issue(s), single-domain antibodies, such as Fab and scFv, have been developed, but these monovalent fragments very often lack the high affinity and long retention times of conventional antibodies. It is therefore common to 'reconstruct' multivalent fragments into bi- and tri-specific Fabs or scFV diabodies or triabodies (see Holliger and Hudson, 2005).

A relatively new addition to the single-domain ligand therapeutic agents is the so-called nanobodies, VHH-based immunoglobulin single-variable domains of heavy-chain antibodies that naturally occur in the Camelidae family. In a recent study by Jähnichen et al. (2010), nanobodies were generated to the extracellular domain of the chemokine receptor CXCR4 (receptor nomenclature follows Alexander et al., 2011). The authors described two different nanobodies that bind to distinct, but partially overlapping, target sites in the extracellular loops. Individually, these nanobodies bound the receptor with reasonable affinity, but coupling them together with linkers ranging from 15 to 20 amino acids resulted in up to a 27-fold increase in apparent affinity for CXCR4. The authors showed that linking one of the active nanobodies to an inactive nanobody decreased the apparent affinity, showing that the linker itself did not contribute to the increased affinity. It also suggests that adding the linker caused some steric hindrance or rotational freedom of the single nanobody. A positive cooperative effect of the two active nanobodies was ruled out by equimolar mixing of the monomers without the linker. It is clear then that the enhanced apparent affinity of the nanobodies was a result of generating biparatopic/heterobivalent 'diabodies' and the associated avidity.
Several groups are taking advantage of the increase in functional affinity offered by linking nanobodies by generating higher-order multimers. For example, Zhang et al. (2004) have generated pentamers of a single-domain nanobody raised against parathyroid hormone (PTH). While the monomer bound immobilized PTH with a $K_{\mathrm{D}}$ of $7 \mu \mathrm{M}$, they found that the pentameric form showed an affinity gain of three to four orders of magnitude. This was accompanied by a corresponding decrease in the dissociation rate constant, falling from over $1 \mathrm{~s}^{-1}$ to an estimated $0.0001 \mathrm{~s}^{-1}$.

Persistent rebinding of a multivalent ligand can lead to its accumulation in the region of its target. This is perhaps best demonstrated by studying the location of antibodies in a tumour, where it is often observed that they have a heterogeneous, largely perivascular distribution (Baker et al., 2008; Rudnick and Adams, 2009). In a study that modelled the penetration of the vascular wall and antigen-antibody interaction, binding of the antibody to antigen retained the ligand in the local area around the target, preventing further diffusion into the tumour (Fujimori et al., 1989), a phenomenon that has been termed the 'binding site barrier' (Weinstein et al., 1987). This relationship between antibody and antigen distribution has since been demonstrated in vivo using autoradiography and immunohistochemistry (Juweid et al., 1992). In these situations, antibody rebinding and accumulation is thought to be highly dependent on the density of its target; thus a high density of antigen can lead to the retention of even low-affinity antibodies (Zuckier et al., 2000).

Although these principles are far more widely utilized for biological therapeutic agents, there is a growing number of examples of bivalent low molecular weight ligands and peptides. The search for improved receptor subtype selectivity has driven the generation of a series of bivalent muscarinic $\mathrm{M}_{2}$ receptor ligands (Disingrini et al., 2006; Steinfeld et al., 2007; Antony et al., 2009). These ligands comprise a fragment that binds the orthosteric, ACh binding site linked to an allosteric fragment that binds to an alternative location on the same receptor. Binding to the orthosteric site is considered to give good affinity, while binding to the allosteric site confers receptor subtype selectivity, although it is interesting to note that the combined binding domain ligand (THRX160209) had a significantly improved affinity and selectivity when compared with the single monomeric components (Steinfeld et al., 2007). In addition, based on the results obtained when performing 'infinite dilution' dissociation experiments, the authors also concluded that this divalent ligand displayed moderately increased residence time when it was simultaneously bound to both sites at the $\mathrm{M}_{2}$ receptor, relative to residence times when only a single site was involved (Steinfeld et al., 2007).

More recently, Steinfeld et al. (2011) reported the discovery of THRX-198321, a bifunctional muscarinic receptor antagonist and $\beta_{2}$-adrenoceptor agonist. This molecule consists of a muscarinic antagonist moiety linked by a C9 polymethylene linker to a $\beta_{2}$-adrenoceptor agonist moiety. They found that THRX-198321 bound with very high affinity to both the $\mathrm{M}_{3}$ muscarinic receptor and the $\beta_{2}$-adrenoceptor, greater than 300-fold that of the monovalent fragments at their cognate receptors. By measuring the effect on orthosteric radioligand dissociation rates, they suggested that the 
ligand binds to both the orthosteric and an allosteric site of both the receptors. Further detailed studies are required to find out whether allosteric enhancement of orthosteric affinity also contributed in some way, but the example shows that by anchoring a second low-affinity ligand close to its binding pocket, the increased local concentration can significantly enhance the propensity to bind.

The rebinding concept has been invoked in several studies of long-lasting binding and avidity of di-/multivalent antibodies and other ligands (Pack et al., 1995; Shinohara et al., 1997; Müller et al., 1998; Todorovska et al., 2001; Arranz-Plaza et al., 2002; Dwir et al., 2003; Gregory et al., 2006; Zichi et al., 2008), but it is not always clear what mechanism is actually referred to in those articles. This illustrates the semantic confusion that still exists around the 'rebinding' terminology. Indeed, this term was initially (and sometimes still is) used in the context of washout-type dissociation experiments to designate the establishment of a new macroscopic binding equilibrium (De Meyts et al., 1973; Limbird and Lefkowitz, 1976). Central to this definition is the assumption that dissociated ligand molecules immediately distribute throughout the new wash-out medium. However, 'rebinding' is now also used to describe phenomena that take place at the micro-anatomic scale, namely the consecutive binding of the same ligand to its target (or those nearby) before it finally escapes into the bulk of the solution. As reviewed elsewhere (Vauquelin, 2010; Vauquelin and Charlton, 2010), this process is favoured when the free threedimensional (3D) diffusion of the dissociated ligand is hindered. This can already be brought about by the cell membrane itself by virtue of its ability to create an adjacent 'unstirred layer' (Berg and Purcell, 1977; De Meyts et al., 1977; Goldstein and Dembo, 1995). Even more hindrance occurs when the ligand is liberated in small membrane-surrounded cavities such as neuronal synapses and other interstitial spaces in tissues (Coombs and Goldstein, 2004; Hrabctová and Nicholson, 2004; Spivak et al., 2006) and for ligands that approach and leave their target via two-dimensional (2D) diffusion within the plane of the cell membrane (Szczuka et al., 2009; Vauquelin and Packeu, 2009).

A situation of avidity-related forced proximity may be considered to represent an extreme form of hindered ligand diffusion and its associated rebinding is therefore likely to be much more profound. To provide better insight into this issue, the simulations presented here explored the magnitude by which forced proximity - and a more mundane 'unstirred layer' - type hindered diffusion may affect a ligand's dissociation behaviour and the manner by which its apparent affinity evolves with time. These simulations (see Supporting Information Appendix S1 for methodology) give an account of the binding of mono- and bivalent ligands to wellseparated target sites on a membrane surface and the consequent effect of hindered diffusion (Figure 1.1, 1.2) as well as the binding of heterobivalent ligands to sufficiently proximate target sites to permit the simultaneous binding of both pharmacophores to their cognate site (Figure 1.3). When comparing the practical outcomes of both mechanisms, the simulations shed light on similarities such as extended target occupancy and the emergence of steep saturation binding curves and, quite strikingly, on the opposing modulation of the ligand's (apparent) affinity.
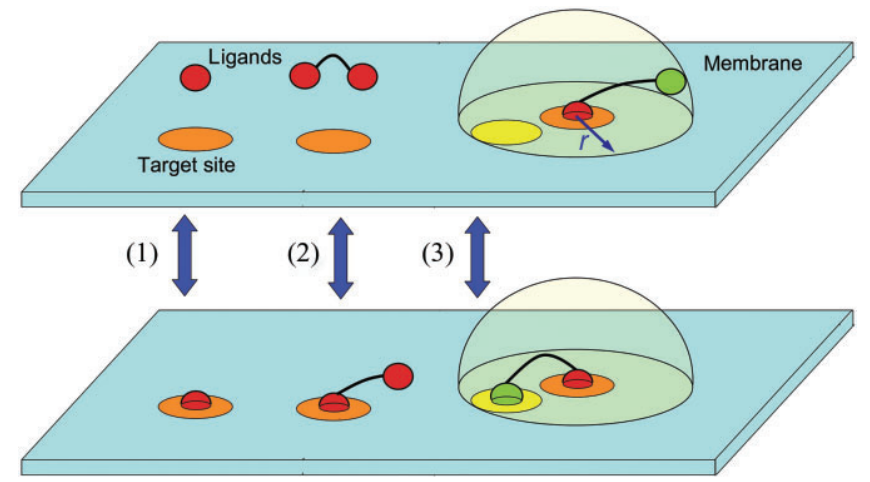

Figure 1

$(1,2)$ Interaction between a monovalent ligand and a bivalent ligand with identicaLpharmacophores (red spheres) and a single isolated target site (orange oval) located at the surface of a cell membrane. Such ligands are further abbreviated as ' $a$ ' and 'aa', respectively, and Figures 7 and 8 depict their binding profiles in situations where their 3D diffusion is either unaffected or hindered. (3) Distinct target sites are present as isolated pairs ' $A B^{\prime}$ ' and are in sufficiently close proximity to be occupied simultaneously by a heterobivalent ligand with matching pharmacophores ' $a b$ '. After the binding of one pharmacophore to its cognate target site, the interaction between the second pharmacophore (green sphere) to its target site (yellow oval) is favoured by their 'forced' proximity. Figures 2-6 depict their binding profiles in different situations.

\section{Nomenclature and description of the models}

Two types of terminologies are generally used in the present context: while bi-/multivalent antibodies bear binding domains that interact with epitopes, synthetic bi-/ multivalent ligands bear pharmacophores that interact with target sites. We will follow the recent, excellent review article by Valant et al. (2012) and use only the latter terminology here. The term 'functional affinity' has also been used by some to designate a bi-/multivalency-related increase in apparent affinity. As this term is a synonym of 'avidity' and also to avoid potential confusion with 'functional selectivity' (a term that is associated with the stabilization of unique receptor conformations, allowing agonists to trigger distinct signalling mechanisms; Kenakin, 2009; Valant et al., 2012), only the term 'avidity' will be used.

For the three models that we have examined here, each ligand/pharmacophore-target site interaction was considered to be a simple, reversible bimolecular process obeying the law of mass-action. Such interaction is sufficient to simulate the binding of a monovalent ligand ' $\mathrm{a}$ ' to its target site ' $\mathrm{A}$ ' (Scheme 1.1). The rates of complex formation and dissociation are governed by the constants $k_{1}$ and $k_{-1}$. A homobivalent ligand ' $a a^{\prime}$ possesses two equivalent pharmacophores ' $a$ ' and here it is assumed that the individual target sites ' $\mathrm{A}$ ' are so far apart that only one of its pharmacophores can bind any time (Scheme 1.2). As both pharmacophores are equivalent, the bivalent ligand has twice the probability to undergo successful binding to the target site than that of the monovalent ligand. 
(1)

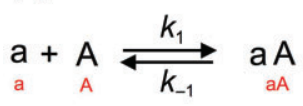

(2)

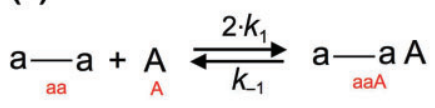

(3)

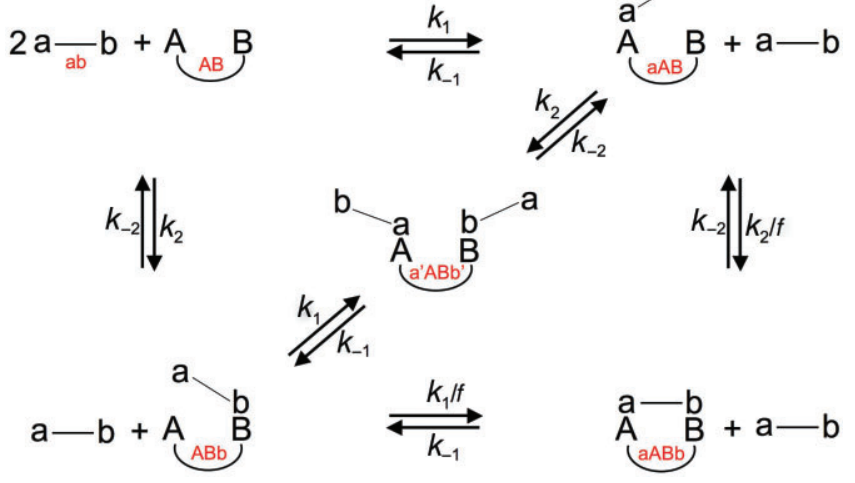

\section{Scheme 1}

Schematic representation of the ligand-target site interactions shown in Figure 1. The abbreviated notation for the molecular species and complexes is in red. ' $a$ ' is a monovalent ligand in (1), ' $a a^{\prime}$ is a bivalent ligand with tethered, equivalent pharmacophores in (2) and ' $a b^{\prime}$ ' is a heterobivalent ligand with distinct pharmacophores ' $a$ ' and ' $b$ ' in (3). ' $A$ ' is a single isolated target site in (1) and (2), and 'AB' are the paired proximate target sites (further denoted as target-pair) in (3). $k_{1}$ and $k_{2}$ are the association (in $\cdot \mathrm{M}^{-1} \cdot \mathrm{min}^{-1}$ ) and $k_{-1}$ and $k_{-2}$ dissociation rate constants (in $\cdot \mathrm{min}^{-1}$ ) of the bimolecular interactions taken in consideration and $f$ is a 'penalty' factor for the second pharmacophoretarget site association. Differential equations that govern how the concentration of these different species evolves with time are provided in Supporting Information Appendix S1.

For the bivalency model presented in Scheme 1.3, the ligand ' $\mathrm{ab}$ ' is heterobivalent: it bears two distinct pharmacophores denoted as ' $a$ ' and ' $b$ '. To simulate avidity, the corresponding target sites, ' $\mathrm{A}$ ' and ' $\mathrm{B}$ ', are assumed to be apart by less than the maximal distance, $r$, between ' $a$ ' and ' $b$ ' (Scheme 1.3). This implies that both pharmacophores can bind their cognate target sites simultaneously; ' $\mathrm{A}$ ' and ' $\mathrm{B}$ ' are thus likely to be physically connected to constitute a 'targetpair' 'AB'. Unhindered $\mathrm{a}-\mathrm{A}$ and $\mathrm{b}-\mathrm{B}$ interactions take place with the respective kinetic constants $k_{1}$ and $k_{2}$ for association and $k_{-1}$ and $k_{-2}$ for dissociation. The individual binding events were described by a model (Scheme 1.3) that is based on earlier ones (Kaufman and Jain, 1992; Plückthun and Pack, 1997), but with extensions. Those initial models stipulate that, after the partial binding of the bivalent ligand via one of its pharmacophores (formation of either $\mathrm{aAB}$ via a-A interaction or $\mathrm{ABb}$ via $\mathrm{b}-\mathrm{B}$ interaction), the second tethered pharmacophore is confined to half of a sphere with radius $r$. When ' $A$ ' and ' $B$ ' reside at the surface of a membrane, the local concentration of this second pharmacophore, [L], corresponds to that of one molecule in a volume equal to $\left(2 \cdot \pi \cdot r^{3}\right) / 3$. It is therefore likely to be much higher than the ligand concentration, [ab], in the bulk of the solution. We have extended those models by allowing the rate constant of this second pharmacophore-target site interaction to be decreased by a penalty factor, $f$, accounting for, for example, limited rotational freedom of this pharmacophore. This extension was inspired by recent binding studies by Jähnichen et al. (2010) where the apparent affinity of an 'active' nanobody decreased when coupled via 15-20 amino acids linkers to an 'inactive' nanobody. This observation even indicated that merely linking two nanobodies via a peptide chain may already cause some steric hindrance or loss of rotational freedom for the initial binding event. An additional extension accounted for the ability of another free ligand to bind when one of the target sites is still vacant (i.e. to yield the hybrid $\mathrm{a}^{\prime} \mathrm{ABb}$ ' species). Only the overall occupancy of ' $\mathrm{AB}$ ' (i.e. $[\mathrm{AB}]_{\mathrm{occ}}$, corresponding to the sum of all bound species, see equation 10 in Supporting Information Appendix S1) is considered for the present simulations. In this respect, aABb was found to constitute by far the most abundant species and it is only under highly unfavourable conditions for its formation (i.e. a high value of $r, f$ and [ab], especially in combination) that some other bound species started to represent a significant fraction (data not shown).

This bivalency model represents the simplest situation in which binding of divalent ligands may result in a net increase in apparent affinity and residence time. Moreover through the use of differential equations, the present simulations allow, for the first time, a description of the binding behaviour of such ligands under realistic (i.e. non-equilibrium) experimental conditions. Many variants of this model are likely to exist and their number is probably only limited by our imagination. In this respect, the supplementary ability of both pharmacophores to influence each other's binding characteristics in an allosteric fashion has recently been reviewed by Valant et al. (2012). In that situation, the ligand is referred to as 'bitopic'. Even more intricate situations, such as when one of the pharmacophores displays agonist characteristics, were evoked as well. While such more elaborate models are certainly worth studying by a related approach in the future, they remain beyond the scope of the present analysis. Moreover, to reduce the number of input parameters, we here only explored the situation where the naïve $\mathrm{a}-\mathrm{A}$ and $\mathrm{b}-\mathrm{B}$ binding events are governed by the same association and dissociation rate constants. In practice, such constants are likely to be different, especially when both pharmacophores display distinct physicochemical characteristics.

Finally, to simulate the effect of a distinct ligand ' $c$ ' on the dissociation profile of ' $a$ ', ' $a a^{\prime}$ ' and ' $a b$ ', it is assumed that ' $c$ ' only binds to ' $\mathrm{A}$ ' in a reversible and competitive fashion with the kinetic constants $k_{3}$ and $k_{-3}$. In the case of target-pairs, the c-A interaction is assumed to leave the b-B interaction undisturbed and vice versa. The relevant interactions are presented in Scheme 2.1-2.3.

\section{Forced proximity and avidity}

Simulations were based on the differential equations provided in Supporting Information Appendix S1. As shown in the left panels of Figure 2, the saturation curves of monovalent ligands ' $a$ ' and of bivalent ligands with equivalent pharmacophores ' $a a^{\prime}$ are sigmoidal (when their free concentration 
(1),(2)

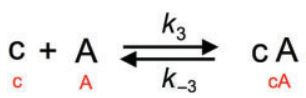

(3)

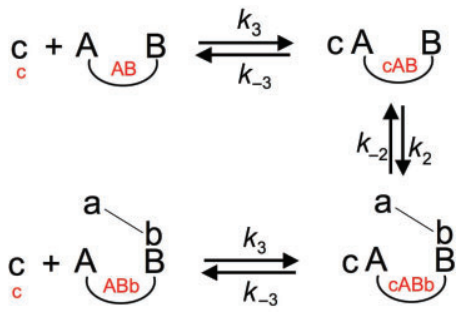

\section{Scheme 2}

Schematic representation of the additional interactions between ligand ' $c$ ' and isolated target sites (1 and 2) and target-pairs (3). Definitions in the legend of Scheme 1 apply and the abbreviated notation for the molecular species and complexes is in red. ' $C$ ' interacts in a competitive manner with ' $A$ ' with association and dissociation rate constants $k_{3}\left(\right.$ in $\cdot \mathrm{M}^{-1} \cdot \mathrm{min}^{-1}$ ) and $k_{-3}\left(\right.$ in $\cdot \mathrm{min}^{-1}$ ) respectively. This interaction is unaffected by the occupancy of ' $\mathrm{B}^{\prime}$ ' by the heterobivalent ligand and vice versa. Differential equations that govern how the concentration of these different species evolves with time are provided in Supporting Information Appendix S1.

is expressed in a logarithmic scale) with a Hill coefficient close to unity (i.e. $n_{\mathrm{H}} \leq 1.02$ ) after $60 \mathrm{~min}$ of incubation when the target sites are far apart and when diffusion is unhindered. The ligand concentrations at which $50 \%$ of the target sites are occupied in this type of simulations closely fit with the theoretical $K_{\mathrm{D}}$ of the individual bonds (defined as $k_{-1} / k_{1}$ ) for the monovalent ligand and half this value for the divalent ligand. With two equivalent pharmacophores, the bivalent ligand is indeed able to associate twice as fast as the monovalent one. Hence, its overall association rate equals $2 \cdot k_{1}$.

Quite distinct binding behaviour is observed for the heterobivalent ligands ' $a b$ ' whose pharmacophores, ' $a$ ' and ' $b$ ', are able to bind simultaneously to their cognate target sites ' $\mathrm{A}$ ' and ' $\mathrm{B}$ ' (Figure 1.3). As shown in the left panels of Figure 2, the saturation binding curves of such ligands show continuing leftward shifts with time and well beyond the initial $60 \mathrm{~min}$. For the sake of simplicity, a-A and b-B binding events were considered to proceed with the same kinetic constants (so that only $k_{1}$ and $k_{-1}$ need to be taken into consideration). A comparison of panels $\mathrm{A}$ and $\mathrm{C}$ reveals the predominant influence of $k_{-1}$ on this delay. Indeed, despite a 10 -fold drop in $k_{-1}$, quasi-equilibrium binding of the heterobivalent ligand is attained after about $2 \mathrm{~h}$ in panel A, whereas it takes almost 8 days in panel C. These and related simulations (not shown) indicate that the time required to achieve quasi-equilibrium binding with such ligands increases in step with the square of the dissociation half-life (further referred to as $t_{1 / 2}$ ) of an individual bond. The panels on the right hand side of Figure 2 provide insight on how the apparent affinity (further denoted as ${ }^{\text {app }} K_{\mathrm{D}(\mathrm{ab})}$ ) and the $n_{\mathrm{H}}$ values of the heterobivalent ligands evolve over time for each of the input $k_{-1}$ values.

When quasi-equilibrium is finally reached, the heterobivalent ligand's affinity is substantially higher than that of the corresponding monovalent ligand. This gain in affinity amounts to $K_{\mathrm{D}(\mathrm{a})} / K_{\mathrm{D}(\mathrm{ab})}$ of 200 (panel A), 500 (panel B) and 2000 (panel C). As further illustrated below, this gain is proportional to $t_{1 / 2}$ and can thus become substantial when the individual bonds are already dissociating slowly by them- selves. However, comparing the affinity of mono- and heterobivalent ligands has only theoretical significance as it is highly unrealistic to obtain $K_{\mathrm{D}(\mathrm{ab})}$ values experimentally because of the often very long incubation times needed. To present a more pragmatic picture, the subsequent simulations will only explore the influence of different binding parameters on the avidity-mediated gain in apparent affinity after a fixed 60 min incubation. Among the different possible graphical representations of such gains, we opted to express the gain as ${ }^{\mathrm{app}} \mathrm{p} K_{\mathrm{D}(\mathrm{ab})}-\mathrm{p} K_{\mathrm{D}(\mathrm{a})}$ and to plot it as a function of $\log \left(t_{1 / 2}\right)$ for visual simplicity in the following Figures.

Figure 3 shows the influence of different $k_{1} / k_{-1}$ combinations on this gain. In this respect, it is of interest to observe first how ${ }^{\mathrm{app}} \mathrm{p} K_{\mathrm{D}(\mathrm{ab})}$ and $\mathrm{p} K_{\mathrm{D}(\mathrm{a})}$ are influenced by the $k_{1} / k_{-1}$ combinations separately. The monovalent ligand saturation curves in Figure $3 \mathrm{~A}$ reflect quasi-equilibrium (i.e. $n_{\mathrm{H}} \leq 1.02$ ) for all the combinations examined and, in agreement with this, $\mathrm{p} K_{\mathrm{D}(\mathrm{a})}$ increases in step with $\log \left(t_{1 / 2}\right)$, with a slope factor of 1 . In contrast, the shape of the corresponding curves for the bivalent ligands is more complex. As shown in Figure 3B, ${ }^{\mathrm{app}} \mathrm{p} K_{\mathrm{D}(\text { (ab) }}$ increases initially also in step with $\log \left(t_{1 / 2}\right)$, but with a slope factor of 2 . Moreover, when $\log \left(t_{1 / 2}\right)$ is further increased, the concomitant increase in ${ }^{\mathrm{app}} \mathrm{p} K_{\mathrm{D}(\mathrm{ab})}$ will progressively slow down and finally come to a halt; ${ }^{\text {app }} \mathrm{p} K_{\mathrm{D}(\mathrm{ab})}$ has then attained an upper limit. Increasing $k_{1}$ causes this upper ${ }^{\mathrm{app}} \mathrm{p} K_{\mathrm{D}(\mathrm{ab})}$ value to increase proportionally and it also allows this value to be attained at lower $\log \left(t_{1 / 2}\right)$ values (Figure 3B). This plateau is indicative of a non-equilibrium situation that results from the limited incubation time. Indeed, while corresponding $n_{\mathrm{H}}$ values are close to unity for the ascending part of the curves, the inflection coincides with a sharp increase in $n_{\mathrm{H}}$ (data not shown). In addition, instead of reaching an upper limit, ${ }^{a p p} \mathrm{p} K_{\mathrm{D}(\mathrm{ab})}$ continues to rise linearly with $\log \left(t_{1 / 2}\right)$ when incubations last long enough for quasi-equilibrium binding to be reached (red symbols in Figure 3B in the case of $k_{1}=1 \times 10^{6} \mathrm{M}^{-1} \cdot \mathrm{min}^{-1}$ ). This further supports the proposition that a quasi-equilibrium situation applies only for this initial ascending phase.

As shown in Figure 3C, the avidity-mediated gain in apparent affinity after $60 \mathrm{~min},{ }^{\mathrm{app}} \mathrm{p} K_{\mathrm{D}(\mathrm{ab})}-\mathrm{p} K_{\mathrm{D}(\mathrm{a})}$, exhibits a bell-shaped pattern when plotted against $\log \left(t_{1 / 2}\right)$ for all values of $k_{1}$ examined. First, app $\mathrm{p} K_{\mathrm{D}(\mathrm{ab})}-\mathrm{p} K_{\mathrm{D}(\mathrm{a})}$ increases in step with $\log \left(t_{1 / 2}\right)$, with a slope factor of 1 . This increase will progressively fade and ultimately give way to a decline. This inversion derives from the fact that the $\mathrm{p} K_{\text {Dapp }}$ of the heterobivalent ligand has reached a plateau value (Figure 3B), whereas $\mathrm{p} K_{\mathrm{D}(\mathrm{a})}$ is still able to increase in step with $\log \left(t_{1 / 2}\right)$ (Figure $3 \mathrm{~A})$. The largest difference between ${ }^{\text {app }} \mathrm{p} K_{\mathrm{D}(\mathrm{ab})}$ and $\mathrm{p} K_{\mathrm{D}(\mathrm{a})}$ is thus obtained at the inflection point of the ${ }^{\text {app }} \mathrm{p} K_{\mathrm{D}(\mathrm{ab})}$ versus $\log \left(t_{1 / 2}\right)$ plot. This implies that, because of the limited incubation time, the highest avidity-mediated gain in 'affinity' will be obtained for large $k_{1}$ /moderate $t_{1 / 2}$ combinations.

The next series of simulations were aimed to examine the influence of the maximal distance between the pharmacophores of the heterobivalent ligand, $r$, and the penalty factor for the second free pharmacophore-target site interaction, $f$, on the avidity-mediated gain in apparent affinity after $60 \mathrm{~min}$. Interestingly, $r$ and $f$ are connected mathematically via the inverse relationship between $[\mathrm{L}]$ and $r^{3}$ and by the persistent presence of the quotient $[\mathrm{L}] / f$ in the differential equations. Consequently, multiplying $r$ by the value $x$ 
A

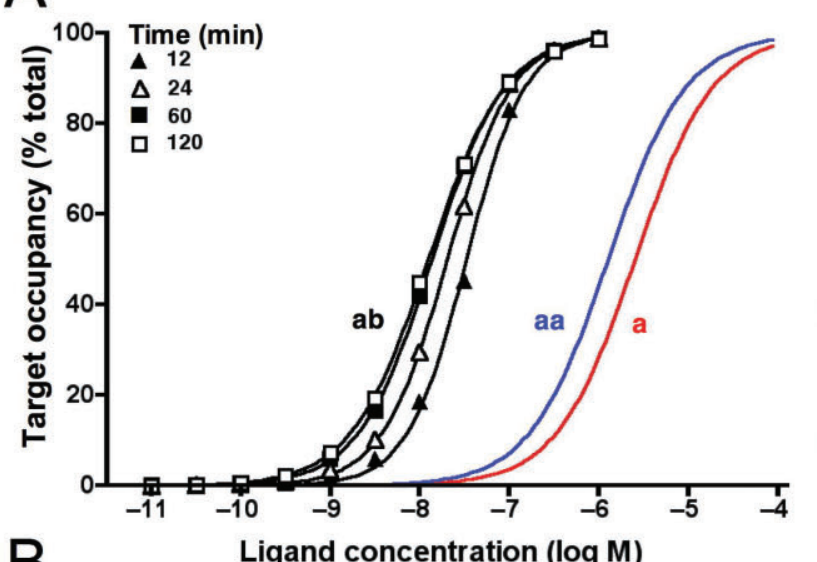

$\mathrm{B} \quad$ Ligand concentration $(\log \mathrm{M})$

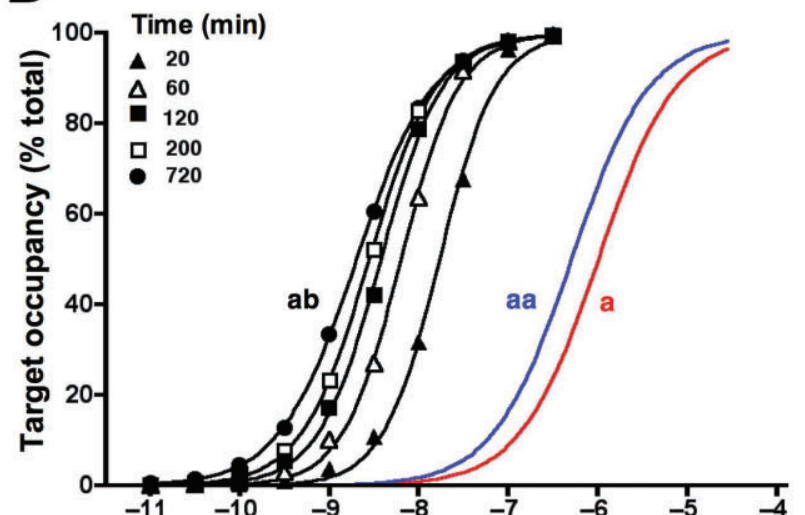

Ligand concentration $(\log \mathrm{M})$

C

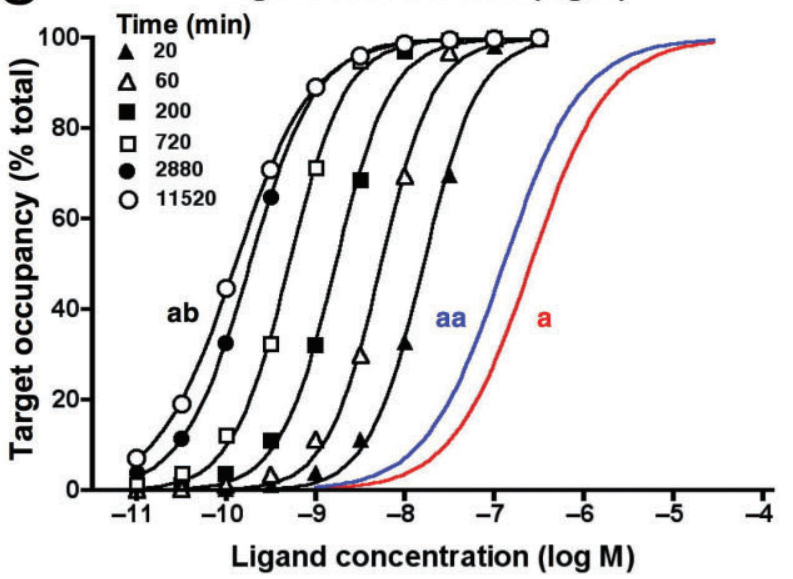

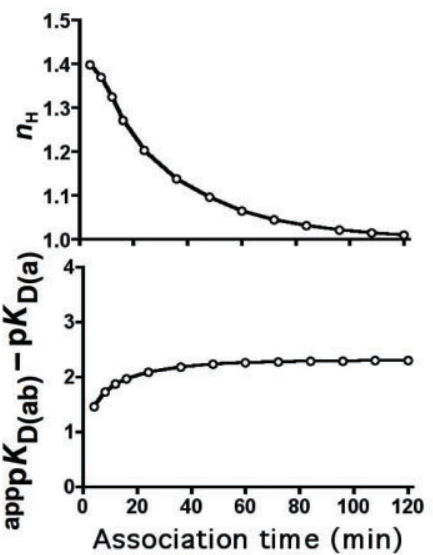
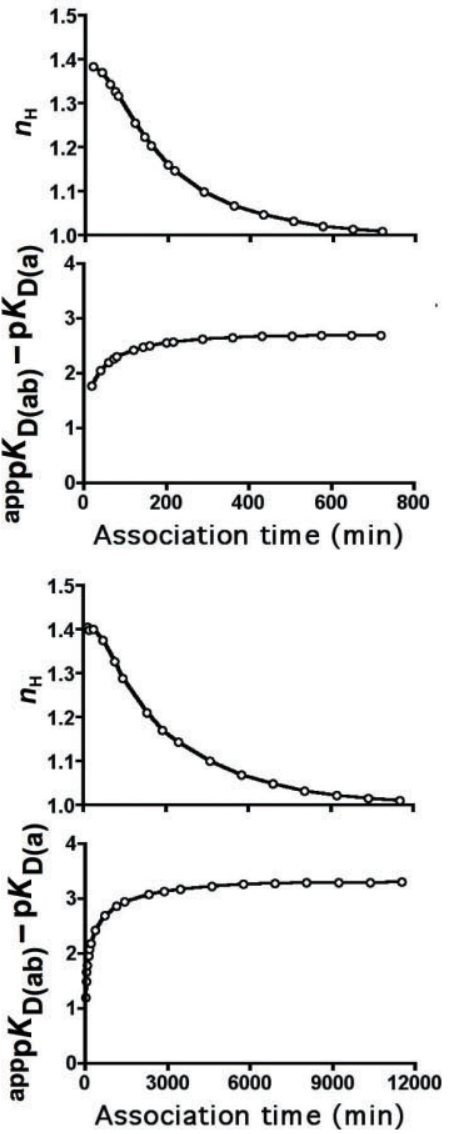

Figure 2

Simulated saturation binding of ' $a$ ' and 'aa' to isolated target sites ' $A$ ' and of 'ab' to target-pairs 'AB' at different incubation times. Left panels: Saturation binding curves at different incubation times (given in each panel). Target site occupancy by 'ab' includes all bound species as enumerated in equation 10 of Supporting Information Appendix S1. Association $k_{1}$ (and $k_{2}$ ) values equal $1 \times 10^{6} \mathrm{M}^{-1}$. $\mathrm{min}^{-1}$ and dissociation $k_{-1}$ (and $k_{-2}$ ) values equal $2.5 \mathrm{~min}^{-1}$ in panel $\mathrm{A}, 1 \mathrm{~min}^{-1}$ in panel B and $0.25 \mathrm{~min}^{-1}$ in panel $\mathrm{C}$. This corresponds to individual bond dissociation $t_{1 / 2}$ values (denoted as $t_{1 / 2}$ further on) of $0.28,0.69$ and 2.8 min respectively. Red curves represent saturation binding of ' $a$ ' under equilibrium conditions (attained at the shortest incubation time); its concentration causing $50 \%$ occupancy (denoted as $K_{\mathrm{D}(\mathrm{a})}$ further on) is $2.5,1$ and $0.25 \mu \mathrm{M}$ in panels $A, B$ and $C$ respectively. Blue curves represent saturation binding of 'aa' under equilibrium (also attained at the shortest incubation time);

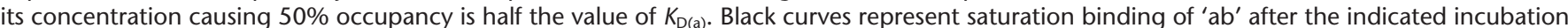
times and with $r=54 \AA$ and $f=10$. Right panels: Analysis of 'ab' saturation binding curves shown in the corresponding left panels. An extended range of association times is examined here. Top: the time-dependent approach to quasi-equilibrium is reflected by the decrease in steepness of the curves (quasi-equilibrium is considered to be attained when $n_{H} \leq 1.02$ ). Bottom: The leftward shift of the 'ab' curves is designated by the quotient between $K_{\mathrm{D}(\mathrm{a})}$ and the apparent $K_{\mathrm{D}}$ values of 'ab' and presented as ${ }^{\text {app }} \mathrm{p} K_{\mathrm{D}(\mathrm{ab})}-\mathrm{p} K_{\mathrm{D}(\mathrm{a})}$. These values increase until a limit (i.e. $2.3,2.7$ and 3.3 in panels $A, B$ and $C$ respectively) is attained. 

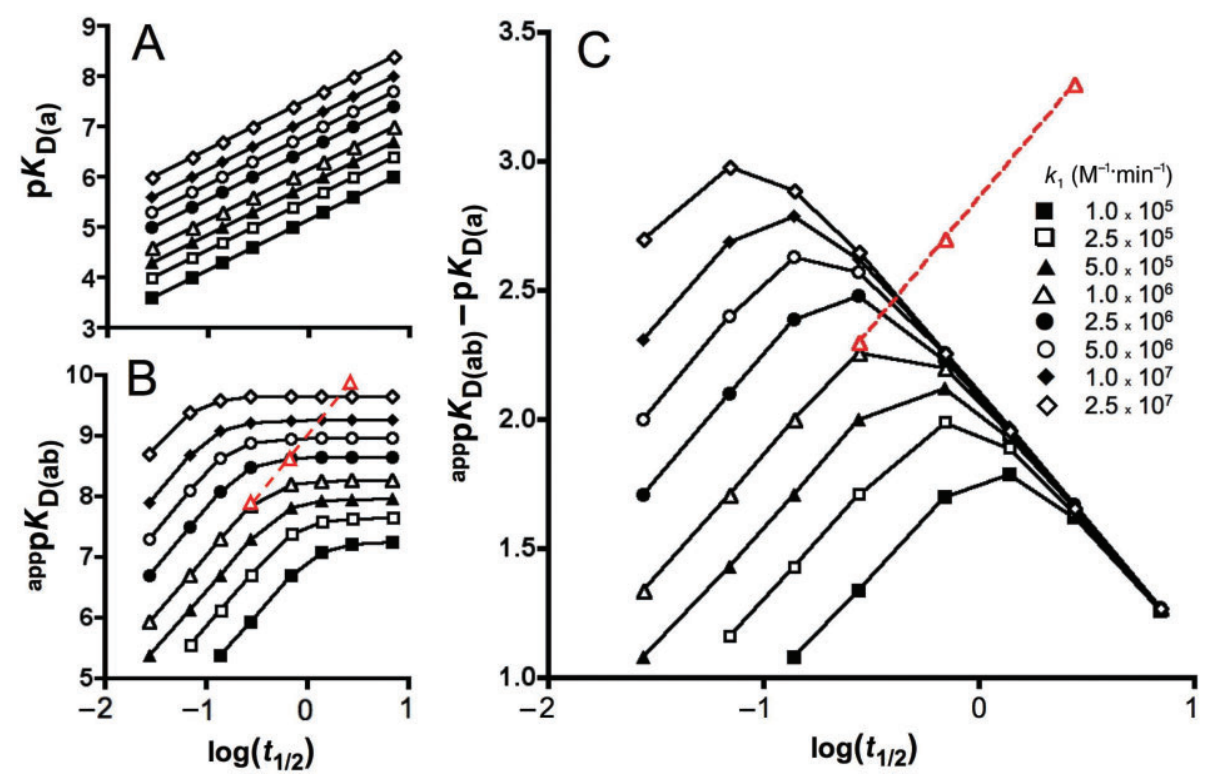

\section{Figure 3}

Simulated $\mathrm{a}-\mathrm{A}$ and $\mathrm{ab}-\mathrm{AB}$ saturation binding: effect of $k_{1}$ and $k_{-1}$ [expressed in the abscissa as $\log \left(t_{1 / 2}\right)$ with $t_{1 / 2}$ in min]. For all ab-AB saturation binding curves: $r=54 \AA$ and $f=10$. Symbols refer to values of $k_{1}$; black symbols correspond to 60 min of incubations and red symbols correspond to quasi-equilibrium binding of 'ab' examined in Figure $2 \mathrm{~A}-\mathrm{C}$. $(\mathrm{A}, \mathrm{B}) \mathrm{p} K_{\mathrm{D}(\mathrm{a})}$ and ${ }^{\mathrm{app}} \mathrm{p} K_{\mathrm{D}(\mathrm{ab})}$ values are presented individually as a function of log(t $\left.t_{1 / 2}\right)$ for the different values of $k_{1}$ disclosed in panel C. (C) ${ }^{a p p} p K_{D(a b)}-p K_{D(a)}$ as a function of $\log \left(t_{1 / 2}\right)$ and for different values of $k_{1}$.

yields the same outcome as multiplying $f$ by $x^{3}$. Hence, there is no need to examine the impact of $r$ and $f$ individually. Although we introduced $f$ to account for phenomena like a limited rotational freedom of a remaining free pharmacophore, it may also act as a cooperativity factor when pharmacophores modulate each other's affinity by changing their association rate. Indeed, $f$ should be inversely related to $\alpha$, the cooperativity factor in the general allosteric ternary complex model (Christopoulos, 2002; Christopoulos and Kenakin, 2002).

As increasing $f$ (or $r$ ) only affects the second pharmacophore-target site interaction in our model (Scheme 1.3), it will not have the same consequences as decreasing $k_{1}$, as examined in Figure 3 . As shown in Figure $4 \mathrm{~A}$, increasing $f$ produces a rightward shift of the ${ }^{a p p} \mathrm{p} K_{\mathrm{D}(\mathrm{ab})}$ versus $\log \left(t_{1 / 2}\right)$ plot without affecting the slope factor (of 2) of the initial ascending section or the upper limit of ${ }^{\text {app }} \mathrm{p} K_{\mathrm{D}(\mathrm{ab})}$ (Figure $4 \mathrm{~A}$ ). As illustrated by the blue arrow in Figure $4 \mathrm{~A}$, this rightward shift reflects a decrease in ${ }^{\mathrm{app}} \mathrm{p} K_{\mathrm{D}(\mathrm{ab})}$ and the associated faster attainment of quasi-equilibrium binding at any given $k_{1} / k_{-1}$ combination. This can be attributed to the fact that $f$ decreases the likelihood of the heterobivalent ligand's second pharmacophore to bind to its cognate target site. Similarly, increasing $r$ will decrease ${ }^{\text {app }} \mathrm{p} K_{\mathrm{D}(\mathrm{ab})}$ as well because of the concomitant decrease in the local concentration [L] of the second tethered ligand. The $\mathrm{p} K_{\mathrm{D}(\mathrm{a})}$ versus $\log \left(t_{1 / 2}\right)$ relationship (in red) is obviously not affected by $r$ and $f$ and is only shown for comparison. As shown in Figure $4 \mathrm{~B}$, the ${ }^{\mathrm{app}} \mathrm{p} K_{\mathrm{D}(\mathrm{ab})}-\mathrm{p} K_{\mathrm{D}(\mathrm{a})}$ versus $\log \left(t_{1 / 2}\right)$ plots display the same bell-shaped pattern as in Figure $3 \mathrm{C}$ and the highest avidity-mediated gain in apparent affinity is obtained for low $f$ (or $r$ )/moderate $t_{1 / 2}$ combinations.

\section{Forced proximity and residence time}

To simulate the dissociation of pre-bound ligands, the washout process was initiated by setting the free ligand concentration to 0 . In experimental terms, this corresponds to replacing the initial ligand-containing medium with a large excess of fresh, naïve medium. For those simulations, we also assumed that, even if the dissociated ligand molecules were distributed throughout this medium, the resulting concentration should be so low that the establishment of a new massaction-type binding equilibrium is negligible. Similar to the situation with monovalent ligands and divalent ligands with equivalent pharmacophores (in the case of well-separated target sites), the dissociation of heterobivalent ligands from target-pairs can be satisfactorily described by a monoexponential dissociation paradigm (Figure 5A). However, while the former dissociate with the same rate (solely dictated by $k_{-1}$ ), full liberation of the target-pairs by the heterobivalent ligands (i.e. recovery of the unbound ' $\mathrm{AB}^{\prime}$ ' species) takes place at a much slower pace (Figure 5A and Table 1). The dissociation half-life of $[\mathrm{AB}]_{\text {occ }}$ is referred to as $\log \left(t_{1 / 2(a \mathrm{ab})}\right)$ and continues to increase unabated and with a slope factor of 2 when plotted against $\log \left(t_{1 / 2}\right)$ (Figure $5 \mathrm{~B}, \mathrm{C}$ ). This implies that the forced proximity-mediated gain in residence time is always proportional to the residence time of an equivalent monovalent ligand. Alternatively, when $\log \left(t_{1 / 2}\right)$ is kept constant, a considerable gain in $\log \left(t_{1 / 2(a b)}\right)$ can also be achieved when increasing $k_{1}$ and/or decreasing $f$ and $r$ (Figure 5B, C). These latter contributions can be explained by the increased likeliness of the partially dissociated complexes (i.e. $\mathrm{aAB}$ and $\mathrm{ABb}$ ) to convert back into the doubly linked one (i.e. $\mathrm{aABb}$ ) rather than dissociating completely. 

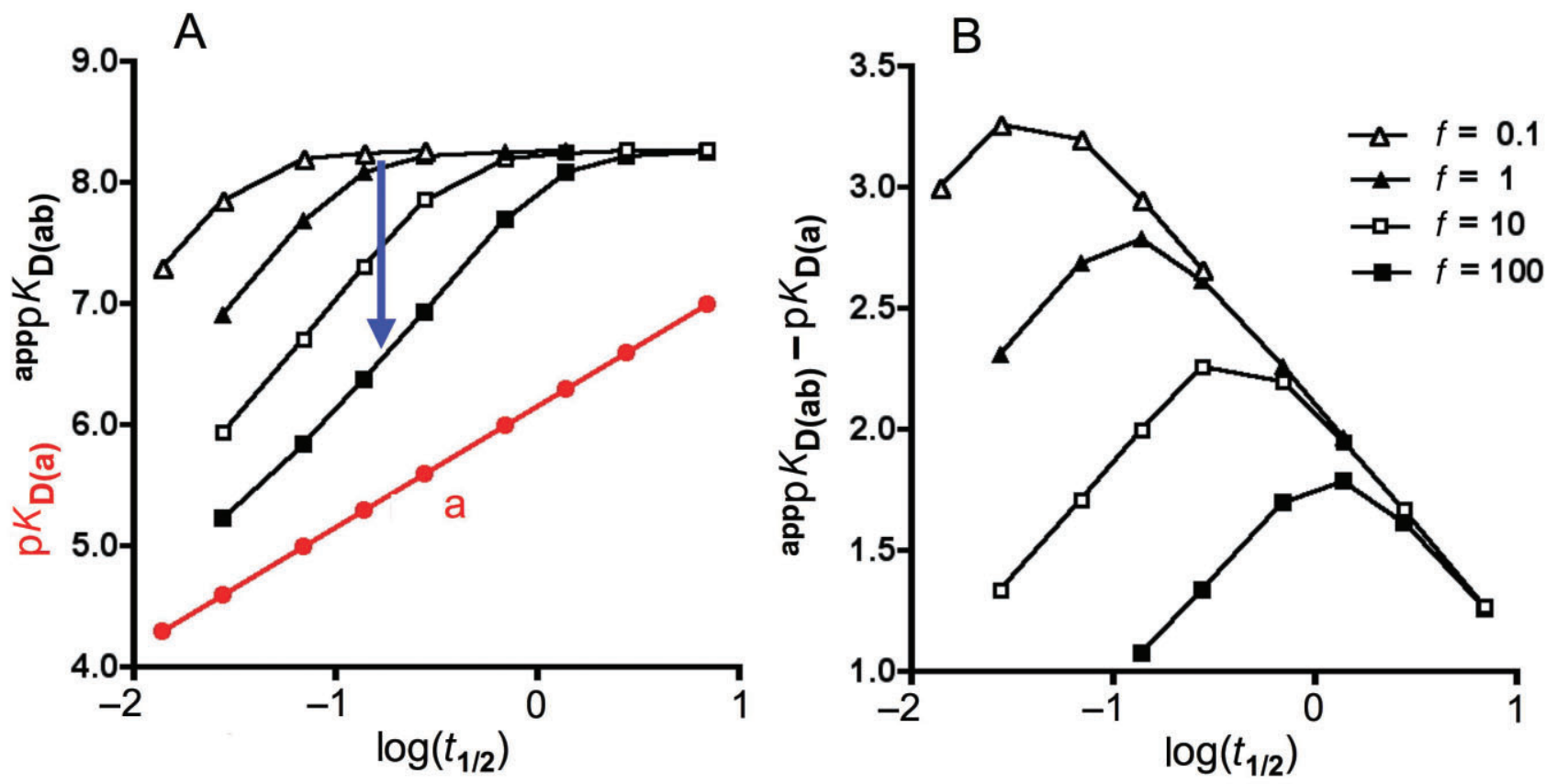

Figure 4

Simulated a-A and ab-AB saturation binding: effect of $f$ and $k_{-1}$ [expressed in the abscissa as $\log \left(t_{1 / 2}\right)$ with $t_{1 / 2}$ in min]. Incubation times were set to $60 \mathrm{~min}, k_{1}=1 \times 10^{6} \mathrm{M}^{-1} \cdot \mathrm{min}^{-1}$ and $r=54 \AA$ for ab-AB interactions. (A) $\mathrm{p} K_{\mathrm{D}(\mathrm{a})}$ and ${ }^{\mathrm{app} p} K_{\mathrm{D}(\mathrm{ab})}$ values are presented as a function of log $\left(t_{1 / 2}\right)$ for the different values of $f$ disclosed in panel $B$. As illustrated by the blue arrow, the rightward shift of these curves results, at constant $k_{-1}$, in a decrease in ${ }^{a p p} p K_{D(a b)}$ and a faster attainment of quasi- equilibrium binding. (B) ${ }^{a p p} p K_{D(a b)}-p K_{D(a)}$ as a function of log $\left(t_{1 / 2}\right)$ and for different values of $f$.

\section{Table 1}

Calculated dissociation $t_{1 / 2}$ values of monovalent and heterobivalent ligand 'AB' complexes ( $t_{1 / 2}$ and $t_{1 / 2(a b)}$ in min, respectively) as a function of $k_{-1}$

\section{$k_{-1}\left(\min ^{-1}\right)$}

$t_{1 / 2}(\min )$

$t_{1 / 2(a b)}(\min )$

\begin{tabular}{llc|}
10 & 0.069 & 1.9 \\
5 & 0.14 & 7.3 \\
2.5 & 0.28 & 29 \\
1 & 0.69 & 180 \\
0.5 & 1.4 & 710 \\
\hline
\end{tabular}

Other parameters are $k_{1}=1 \times 10^{6} \mathrm{M}^{-1} \cdot \mathrm{min}^{-1}, r=54 \AA$ and $f=10$. Simulated data (with examples shown in Figure 5A) were analysed by GraphPad Prism 4.0 (GraphPad Software Inc., San Diego, CA, USA) according to a mono-exponential dissociation paradigm with $0 \%$ occupancy as the lower asymptote.

As already pointed out in the Introduction, a large increase in target residence time can be explained by the fact that a freshly dissociated pharmacophore is forced to remain in 'forced proximity' to its cognate binding site as long as its tethered, companion pharmacophore is still bound. Note that when both pharmacophores display distinct dissociation profiles (which is the most likely situation in case of heterobivalent ligands), the one with the largest dissociation rate constant is likely to be the first one to dissociate. However, as rate constants have only macroscopic/statistical significance, it is also extremely improbable for both pharmacophores to dissociate simultaneously when their dissociation rate constants are identical.

The addition of a competing ligand ' $c$ ' in the washout does not affect the dissociation of freely diffusing monovalent and divalent ligands (in the case of well-separated target sites). Nonetheless, the dissociation of heterobivalent ligands from their target-pairs will be accelerated by such competing ligands even when they only interact with one of the target sites available (Kramer and Karpen, 1998). We also performed simulations to mimic such wash-out conditions, based on the assumption that ' $\mathrm{C}$ ' only binds to ' $\mathrm{A}$ ' in a reversible and competitive fashion and that this interaction leaves the $\mathrm{b}-\mathrm{B}$ interaction undisturbed and vice versa. The relevant interactions are presented in Scheme 2.1-2.3 and the corresponding (new and modified) differential equations are given in Supporting Information Appendix S1. These equations also take account of the existence of $\mathrm{cAB}$ as well as of the hybrid cABb species. Hence, to obtain the overall occupancy of $A B$ by the heterobivalent ligand (i.e. $[\mathrm{AB}]_{\mathrm{occ}}$ ), $\mathrm{cABb}$ must also be included in the sum of bound species (see equation 14 in Supporting Information Appendix S1).

As illustrated in Figure 6A, the time-dependent decrease of $[\mathrm{AB}]_{\text {occ }}$ is accelerated by ' $\mathrm{C}$ ' in a concentration-dependent fashion, but without reaching the dissociation profile of an equivalent monovalent ligand (in red). Indeed, even in the presence of a maximally effective concentration of ' $c$ ', the heterobivalent ligand's dissociation curve will temporarily lag behind that of a freely diffusing mono-/divalent ligand. This is 

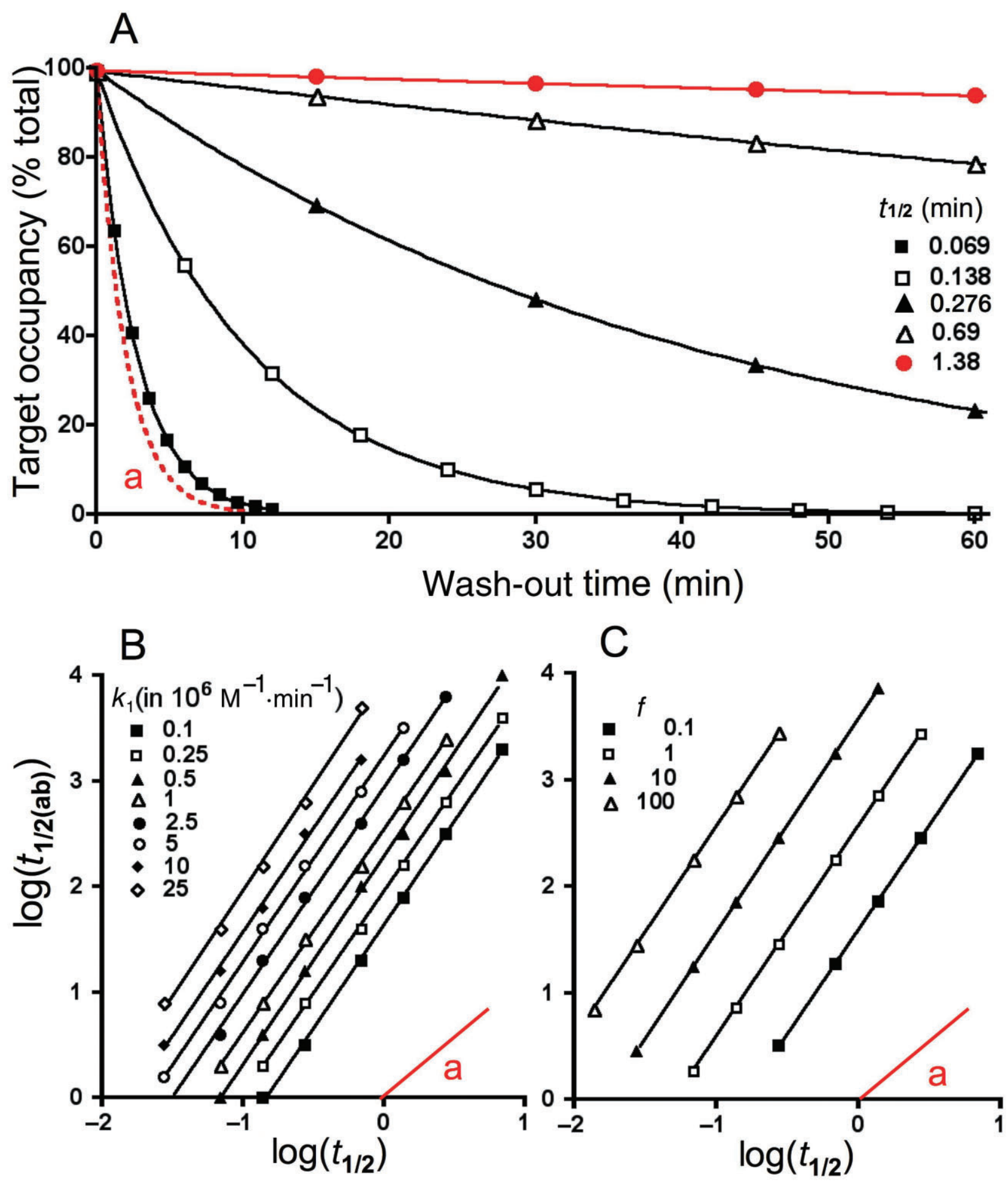

Figure 5

Simulated ab-AB dissociation: effect of $f, k_{1}$ and $k_{-1}$ [expressed in the abscissa as log $\left(t_{1 / 2}\right)$, with $t_{1 / 2}$ in min]. Target site occupancy by 'ab' includes all bound species as enumerated in equation 10 of Supporting Information Appendix $S 1$ and amounts to $99 \%$ of $[A B]_{\text {tot }}$ at the onset of the dissociation. Washout is initiated at $t=0$ by setting [ab] to 0 . Default parameters and units: $k_{1}=1 \times 10^{6} \mathrm{M}^{-1} \cdot \mathrm{min}^{-1}, f=10, r=54 \AA$. (A) Example of time-dependent decline in target site occupancy by 'ab' with different $k_{-1}$ values (solid curves). Curves are generated by a mono-exponential dissociation model with $0 \%$ occupancy as lower asymptote. Red (for the sake of comparison): decline in target site occupancy by 'ab' (solid curve) and by ' $a$ ' with the same $k_{-1}$ value (broken curve). Calculated binding parameters are given in Table 1. (B, C) Dissociation $t_{1 / 2}$ of 'ab', denoted as $\log \left(t_{1 / 2(a b)}\right)$, as a function of $\log \left(t_{1 / 2}\right)$ for different values of $k_{1}$ and $f$ respectively. Red line refers to monovalent a-A dissociation. 

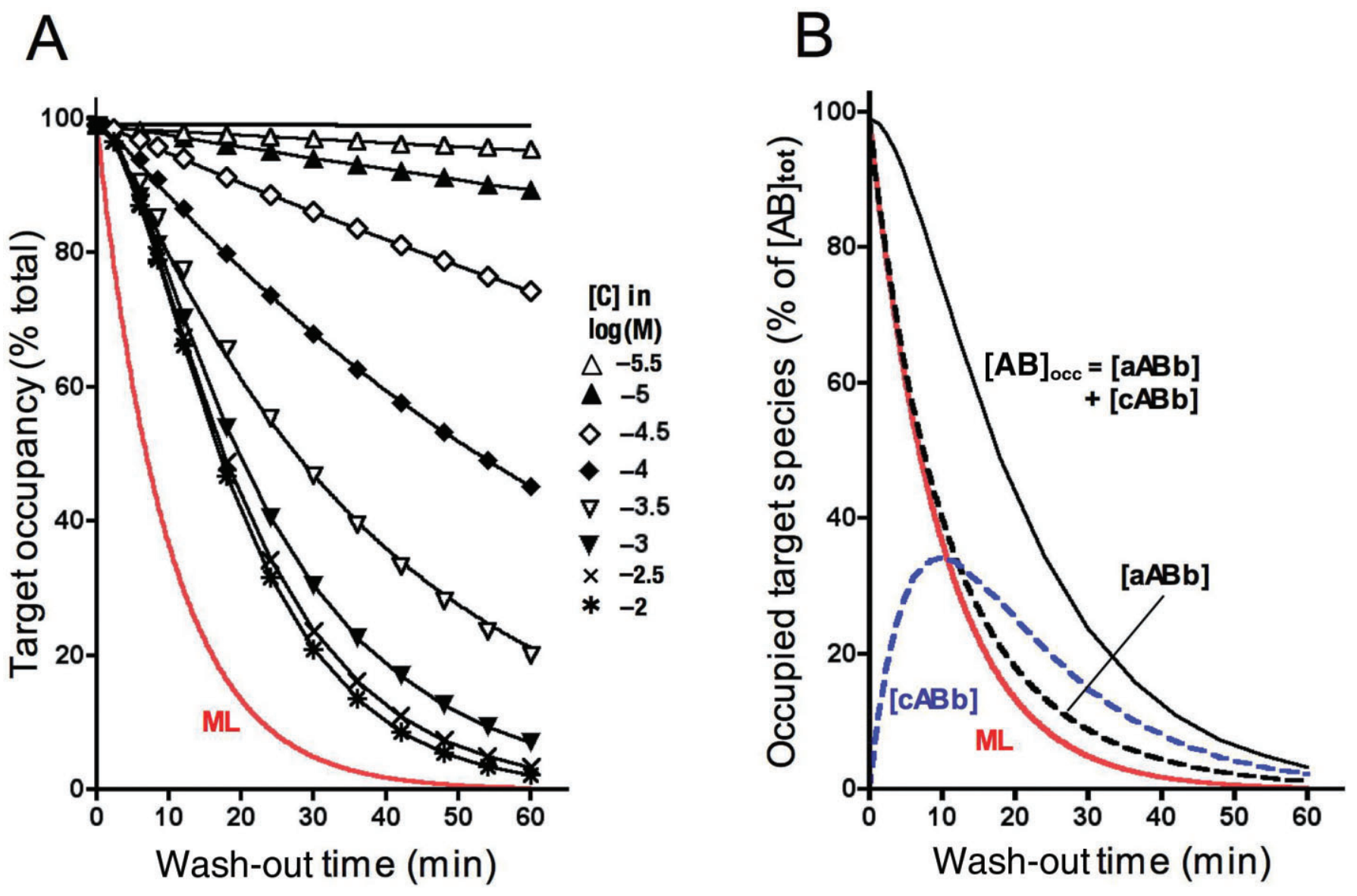

Figure 6

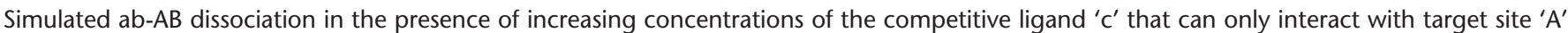
with association $\left(k_{3}\right)$ and dissociation $\left(k_{-3}\right)$ rate constants of $1 \times 10^{7} \mathrm{M}^{-1} \cdot \mathrm{min}^{-1}$ and $1 \mathrm{~min}^{-1}$ respectively. The interaction with ' $\mathrm{c}^{\prime}$ is depicted in Scheme 2 and described by the differential equations in Supporting Information Appendix S1. (A) Black curves: Examples of time-dependent decline in target site occupancy ([AB] $]_{\text {occ, }}$ as defined by equation 14 of Supporting Information Appendix S1) by 'ab' without ' $c$ ' (no symbols) or with the indicated concentrations of ' $c^{\prime}$ ' (symbols). $[A B]_{\text {occ }}$ amounts to $99 \%$ of $[A B]_{\text {tot }}$ at the onset of the washout and the dissociation is initiated by setting [ab] to 0 . Binding parameters: $k_{1}=1 \times 10^{6} \mathrm{M}^{-1} \cdot \mathrm{min}^{-1}, k_{-1}=0.1 \mathrm{~min}^{-1}, r=54 \AA, f=10$. Red curve: Dissociation of ' $\mathrm{a}$ ' with the same $k_{-1}$ value. (B) Time-dependent changes in $[A B]_{\text {occ }}$ and its constituent species. [AB] occ is only composed of [aABb] at the onset of the washout and [cABb] rises transiently as time progresses. Binding parameters are the same as in panel $A$ and $[c]=3 \mathrm{mM}$. Red curve: Dissociation of ' $a$ ' with the same $k_{-1}$ value.

because even if binding of 'c' to liberated target sites proceeds fast enough to prevent all re-association of the original pharmacophore, the dissociation process will only be complete when the tethered companion pharmacophore has dissociated as well. As shown in Figure 6B, this results in a transient existence of the hybrid cABb species in which the target-pair is bound to ' $\mathrm{c}$ ' and also still partially bound to ' $\mathrm{ab}$ '. Bound species other than $\mathrm{aABb}$ and $\mathrm{cABb}$ are negligible both at the onset and during the progress of the washout (data not shown).

The dose-dependency of the accelerating effect by ' $c$ ' after $60 \mathrm{~min}$ of washout is pictured in the inset of Figure 8. The data match a one-site competition binding model, but the calculated $\mathrm{IC}_{50}$ value $(68 \mu \mathrm{M})$ is substantially higher than the theoretical $K_{\mathrm{D}}$ value of ' $\mathrm{c}$ ' $\left(=k_{-3} / k_{3}=0.1 \mu \mathrm{M}\right)$. This marked difference may be explained by the fact that ' $c$ ' needs to be present at very high concentrations to prevent a-A re-association when both are in 'forced proximity' (i.e. a condition where the local concentration of ' $a$ ', $[\mathrm{L}]$, is very high).

\section{Hindered ligand diffusion}

As outlined in detail elsewhere (Vauquelin, 2010; Vauquelin and Charlton, 2010), hindered diffusion will not only induce dissociated ligand molecules to undergo rebinding but it will also delay the association process because of the slower replacement of ligand molecules that were taken away from the vicinity of the membrane by the binding process. The equations to describe these processes have been developed independently in different life science disciplines, but they have the same mathematical form (Perry et al., 1980; Frost and Wagner, 1984; Goldstein and Dembo, 1995; Schuck and Minton, 1996; Christensen, 1997). Particularly the overall/ 
macroscopic association and dissociation events are no longer governed by rate constants but by the rate coefficients, $k_{1} /\left(1+k_{1} \cdot[\mathrm{A}] \cdot k\right)$ and $k_{-1} /\left(1+k_{1} \cdot[\mathrm{A}] \cdot k\right)$ for a monovalent ligand and, because of its twofold higher probability to bind, by the rate coefficients $2 \cdot k_{1} /\left(1+2 \cdot k_{1} \cdot[\mathrm{A}] \cdot k\right)$ and $k_{-1} /\left(1+2 \cdot k_{1} \cdot[\mathrm{A}] \cdot k\right)$ for a homobivalent ligand in the case of well-separated target sites. The parameter ' $k$ ' depends on the diffusion rate of the free ligand and the geometric characteristics of the unstirred layer (Coombs and Goldstein, 2004; Vauquelin and Charlton, 2010). The present simulations focus on the effects of hindered ligand diffusion on the binding properties of monovalent and divalent ligands in the case of well-separated target sites (Scheme 1.1 and 1.2; the corresponding differential equations are given in Supporting Information Appendix S1).

These equations stipulate that increasing the association rate constant, $k_{1}$, should increase the effect of hindered diffusion on the ligand binding properties. This deduction is confirmed by the simulated binding data. When $k_{1}$ is only $1 \times 10^{6} \mathrm{M}^{-1} \cdot \mathrm{min}^{-1}$, this mechanism will only minimally affect the saturation and dissociation binding curves of monovalent and homobivalent ligands. The saturation curves are still sigmoidal after $60 \mathrm{~min}$ of incubation, the bivalent ligand has twice the potency of the monovalent one and both dissociate with nearly the same rate (data not shown). However, when their $k_{1}$ is raised to $1 \times 10^{7} \mathrm{M}^{-1} \cdot \mathrm{min}^{-1}$, hindered diffusion clearly starts to affect the binding characteristics of those ligands, as shown by the rightward shift and steepening of the saturation curves when compared with the free 3D diffusion controls (Figure 7A and Table 2). Closer scrutiny reveals that this mechanism also generates a delay in the attainment of equilibrium binding (Figure $7 \mathrm{~B}$ ). Consequently, the ${ }^{\mathrm{app}} \mathrm{p} K_{\mathrm{D}}$ values (as well as the $n_{\mathrm{H}}$ values, not shown) of such curves need more than $60 \mathrm{~min}$ to reach the theoretical equilibrium values (Figure 7C) and, conforming to the equations shown, this delay is more obvious for the homobivalent ligand.

These equations also explain why hindered diffusion delays the overall dissociation of the bivalent ligand more than that of the monovalent ligand (Figure 8). Here again, such delay only becomes perceptible when $k_{1}$ is raised from 1 $\times 10^{6}$ to $1 \times 10^{7} \mathrm{M}^{-1} \cdot \mathrm{min}^{-1}$ (data not shown). This delay is also more pronounced at low target occupancy (because of the

\section{Table 2}

Effect of ligand bivalency and hindered diffusion on ligand saturation binding to isolated targets

\begin{tabular}{|lll|} 
Ligand & $\begin{array}{l}\mathbf{p} K_{\mathrm{D}} \text { and }\left(\boldsymbol{n}_{\mathbf{H}}\right) \\
\text { for free 3D } \\
\text { diffusion }\end{array}$ & $\begin{array}{l}\text { app } \mathbf{p} \boldsymbol{K}_{\mathrm{D}} \text { and }\left(\boldsymbol{n}_{\mathrm{H}}\right) \\
\mathbf{f o r} \text { hindered } \\
\text { diffusion }\end{array}$ \\
\hline $\begin{array}{l}\text { Monovalent, 'a' } \\
\text { Homobivalent, 'aa' }\end{array}$ & $8.00(1.00)$ & $7.85(1.37)$ \\
\hline
\end{tabular}

Binding data after 60 min of incubation were simulated and the derived binding curves are shown in Figure 7A (binding parameters are given in the legend). The data were analysed by GraphPad Prism 4.0 (GraphPad Software Inc.) according to a variable slope sigmoidal concentration-response paradigm to yield the listed ${ }^{\text {app }} \mathrm{p} K_{\mathrm{D}}$ values and Hill coefficients $\left(n_{\mathrm{H}}\right)$. factor $k_{1} \cdot[\mathrm{A}] \cdot k$ in the denominator) and this property is responsible for an upward inflection of the dissociation curve with time (because more and more targets are liberated). Nevertheless, binding is still decreasing at the nadir in Figure 8 and it will ultimately vanish. Note that the equations for ligand dissociation, under hindered diffusion (Supporting Information Appendix S1), do not describe a mono-exponential decline in ligand binding nor a combination of two or more of such processes. Hence, despite the apparent 'biphasic' character of such dissociation curves, they cannot be adequately analysed in terms of a two-site competition paradigm.
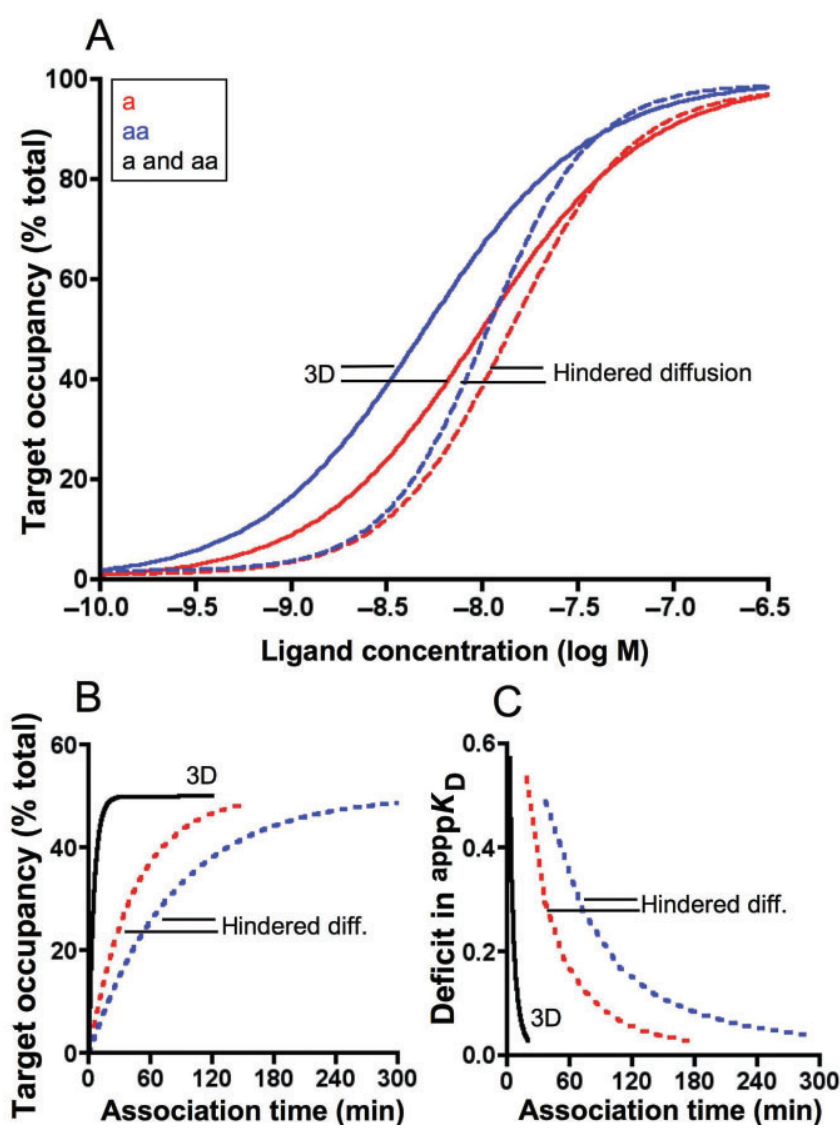

\section{Figure 7}

Simulated occupancy of isolated target sites in the presence of increasing concentrations of ligand: effect of bivalency and hindered diffusion-related rebinding. a-A interactions take place with the rate constants: $k_{1}=1 \times 10^{7} \mathrm{M}^{-1} \cdot \mathrm{min}^{-1}$ and $k_{-1}=0.1 \cdot \mathrm{min}^{-1}$. To simulate hindered diffusion, the product $k_{1} \cdot[\mathrm{A}]_{\text {tot }} \cdot k$ is arbitrarily set to 10 . Curves in black apply when data overlap. (A) Saturation curves of ' $a$ ' and 'aa' after 60 min of incubation in the case of free diffusion (3D) and hindered diffusion. Calculated binding parameters are given in Table 2. (B) Time-dependent increase in ' $a$ ' and ' $a a^{\prime}$ ' binding in case of free diffusion (3D) and hindered diffusion. Ligand concentrations (i.e. 10 and $5 \mathrm{nM}$, respectively) are chosen to yield $50 \%$ target site occupancy at equilibrium. (C) Time-dependent leftward shift of the ' $a$ ' and 'aa' saturation binding curves in the case of free diffusion (3D) and hindered diffusion. Their apparent equilibrium dissociation constants (referred to as ${ }^{a p p} p K_{D}$ ) decrease until the respective equilibrium binding values for ' $a$ ' and 'aa' are finally approached. 


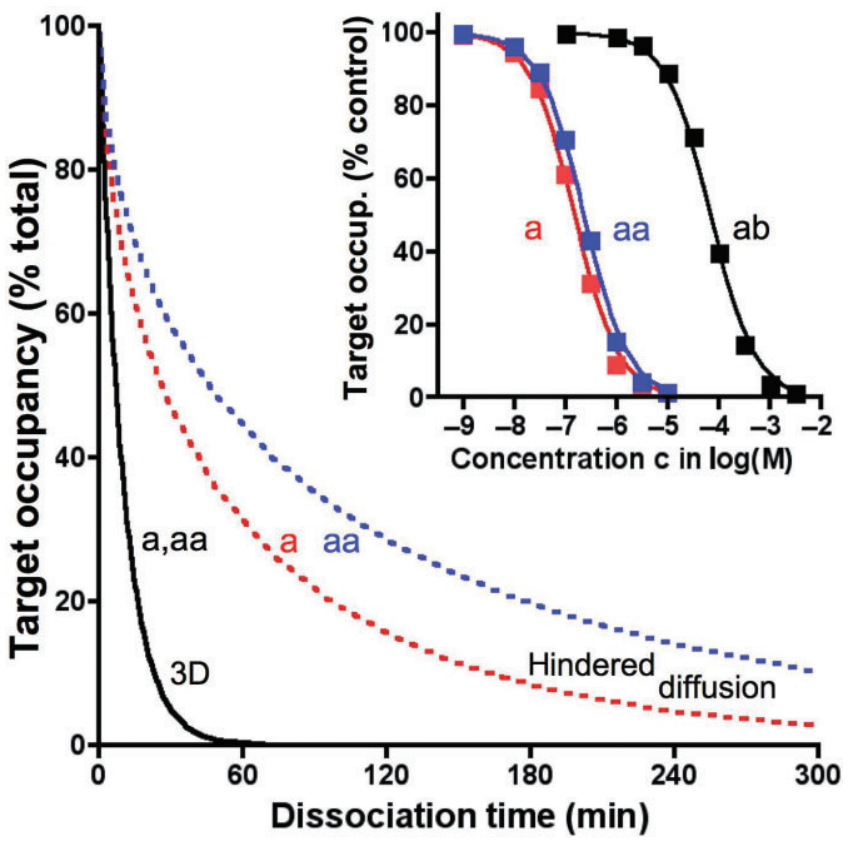

\section{Figure 8}

Simulated a-A and aa-A dissociation in the presence of increasing concentrations of the competitive ligand ' $c$ '. Binding parameters are the same as in Figure 7 for ' $a$ ' and ' $a a^{\prime}$ ' and as in Figure 6 for ' $c$ '. (Main panel) Examples of time-dependent decline in target site occupancy by ' $a$ ' and 'aa' in medium only in the case of free diffusion (3D) and hindered diffusion (with $k_{1} \cdot[\mathrm{A}]_{\mathrm{tot}} \cdot k=10$ ). $99 \%$ of the target sites are bound at the onset of the washout and the dissociation is initiated by setting [a] or [aa] to 0 . Curves in black apply when data overlap. (Inset) Remaining occupancy (after 60 min washout) of ' $A$ ' by ' $a$ ' and ' $a a^{\prime}$ in the case of hindered diffusion and of 'AB' by 'ab': effect of [c]. Control binding refers to remaining target site occupancy after 60 min of washout in medium only. Data are from an extended range of simulations such as shown in Figure $6 \mathrm{~A}$ for ' $a b^{\prime}$ '. The curves are generated by a single-site competition model.

When the hindered diffusion mechanism is operational, addition of a competing ligand ' $c$ ' to the washout medium will also accelerate the dissociation of the monovalent and homobivalent ligands in a concentration-dependent fashion. When the concentration of ' $c$ ' is sufficiently elevated, the dissociation curves will become monophasic and superimposable on the free 3D diffusion, control curves (data not shown). Similar concentration-dependent effect of unlabelled competing ligands has been observed experimentally when studying their effect on the dissociation of radiolabelled antagonists from different GPCRs in intact cells (Fierens et al., 1999; Packeu et al., 2008; Wennerberg et al., 2010). The inset of Figure 8 compares the concentration-dependency of the accelerating effect by ' $c$ ' after $60 \mathrm{~min}$ of washout for the different types of ligands we considered. All simulated data match a one-site competition binding model. In the case of hindered diffusion, ' $c$ ' will accelerate the dissociation of monovalent and homobivalent ligands with relatively high potency $\left(\mathrm{IC}_{50}=0.15\right.$ and $0.23 \mu \mathrm{M}$ respectively). These values are only slightly above its theoretical $K_{\mathrm{D}}$ value $\left(=k_{-3} / k_{3}=\right.$ $0.1 \mu \mathrm{M})$.

\section{Comparison of the effect of both mechanisms on ligand binding}

Bi-/multivalent ligands experience markedly increased affinity and target residence time when at least two of their pharmacophores are able to bind simultaneously to physically linked target sites. It is widely accepted that the occurrence of a single bond will cause the unbound but tethered pharmacophore to stay in 'forced proximity' to the target. This second pharmacophore will thereby acquire a very high local concentration, a factor that greatly favours the (re)formation of the second bond (Kaufman and Jain, 1992; Plückthun and Pack, 1997). This process can be considered to represent an extreme form of ligand rebinding, a term that usually refers to the propensity of freshly dissociated molecules to re-associate with their epitope/target because of some impediment that slows down their diffusion away from the target (Coombs and Goldstein, 2004; Vauquelin, 2010; Vauquelin and Charlton, 2010). It is therefore of interest to compare the effect of both mechanisms on the ligand's affinity for its target and its residence time.

The first major outcome of the present study is that forced proximity and hindered diffusion mechanisms will both delay the attainment of equilibrium binding but that only the former will trigger an increase in affinity (i.e. avidity). This is clearly demonstrated by the continuing timedependent leftward shift of the saturation binding curves in the presence of such mechanisms when, in their absence, the corresponding ligands have already reached equilibrium (Figures 2 and 7). However, the causes for these delays are of different origins. While bivalency will only produce a twofold increase in affinity, the addition of a forced proximity mechanism will produce a marked supplementary increase (Figure 2). Saturation binding will consequently need to be studied at very low heterobivalent ligand concentrations and, because of the positive correlation between the association rate of a bimolecular binding process and the ligand concentration, the initial association process will take much longer to reach equilibrium (this is especially so at the lowest ligand concentrations). Slow initial association may thus blunt the observed potency of heterobivalent ligands and this will lead to somewhat counterintuitive behaviour when the incubation times are kept within reasonable limits. Indeed, although the avidity of the bivalent ligands is positively correlated with $k_{1}$ (along with other factors such as a short distance between the ligand's pharmacophores) and the dissociation $t_{1 / 2}$ of the individual bonds, it is shown in Figures 3 and 4 that increasing $t_{1 / 2}$ beyond a certain limit will actually curb any further increase in the avidity of those ligands. As the incubation time is limited by practical considerations, it is therefore likely that most recorded 'avidity' values are only 'apparent'.

In contrast, a hindered diffusion mechanism will not affect the ligand's affinity itself. Instead, it will be responsible for a slower migration of ligand molecules to the target sites so that each binding process will temporarily produce a local deficit of free ligand. As this phenomenon is most prominent at low ligand concentrations, it will produce steep saturation binding curves. As the incubation time is increased, these curves will gradually shift to the left and 
steepen until equilibrium binding is reached (Figure 7). A local deficit will also be produced twice as fast in the case of bivalency and this deficit is likely to reduce the bivalencymediated gain in potency over short incubation times (Figure 7).

The second major outcome is that forced proximity and hindered diffusion mechanisms will both prolong the residence time of the ligands. When a heterobivalent ligand is only coupled to a target-pair via a single bond, the second, tethered pharmacophore has (whether or not it has freshly dissociated) no other option than to stay in 'proximity' to its cognate target site as long as the single bond remains. Compared with a hindered diffusion-related mechanism where the dissociated ligand molecules only briefly remain close to their target sites, this situation will thus pertain for a much longer time. This combination will greatly favour the second pharmacophore-target site interaction, especially for an elevated $k_{1}$ /low $f$ /low $r$ combination. Rebinding is therefore more prevalent with forced proximity than with a hindered diffusion-related mechanism and the overall dissociation process will therefore also exhibit a more substantial delay. Note that hindered diffusion-related rebinding will also proceed faster in the case of bivalency and when the local concentration of free target sites is high. Bivalent ligands will therefore reside somewhat longer at their target sites than their monovalent counterparts (Figure 8) and, as the concentration of free target sites increases with time in washout dissociation experiments, the dissociation curves will show an apparent biphasic pattern. This deviation from the theoretical mono-exponential decline in binding (in the case of free, un-hindered 3D diffusion of the ligand) actually impedes the correct quantification of the ligand's residence time.

Additionally, the present simulations reveal that, for both mechanisms, the rebinding-related increase in residence time can be counteracted by a competitive ligand ' $\mathrm{C}$ ' (Figures 6 and 8). Indeed, when the washout takes place in the presence of ' $c$ ', it will compete with the freshly dissociated ligands for occupancy of the liberated target sites. This allows ' $\mathrm{c}$ ' to accelerate the overall dissociation process in a concentration-dependent fashion. When ' $\mathrm{c}$ ' can only bind to one of the sites of a target-pair (a situation that is likely to take place when the ligand is of heterobivalent nature), it needs to compete with the corresponding pharmacophore (i.e. ' $a$ ' in the present simulations) to prevent its rebinding. However, being tethered, this pharmacophore will stay much longer nearby its target site than in the case of a freshly dissociated ligand molecule whose diffusion is merely hindered. This implies that, at the same concentration, ' $c$ ' will be much less efficient in preventing the rebinding of the tethered pharmacophore. Hence, although ' $c$ ' is effectively able to accelerate the dissociation of a heterobivalent ligand, this will take place with appreciably lower potency than in the simple hindered diffusion situation (Figure 8, inset). Finally, hindered diffusion could potentially also affect the binding of heterobivalent ligands to their target-pairs. However, this mechanism only becomes operational at quite elevated $k_{1}$. It therefore affects the binding properties of the prototype we considered in the previous section (with $k_{1}$ of only $1 \times 10^{6} \mathrm{M}^{-1} \cdot \mathrm{min}^{-1}$ ) much less than the forced proximity mechanism.

\section{Concluding remarks}

Simultaneous binding of the two pharmacophores of a bivalent ligand to their target sites is likely to produce a marked gain in its affinity (i.e. avidity). Earlier mathematical analyses of such models relied on affinity constants of the individual binding events. Consequently, these only allowed the calculation of the bivalent ligand's genuine affinity or, in other words, its potency under equilibrium conditions. However, the present simulations are based on the underlying rate constants and suggest that experimental measurements are likely to yield affinity values that are only apparent and lower than expected. This is because incubation times that are deemed reasonable for the purpose of, for example, generating saturation binding curves are likely to be less than those actually required to reach genuine equilibrium binding. Taking this 'time-pressure' into account, the simulations suggest that the highest gain in apparent affinity is most likely to be obtained when the constituting pharmacophores display large $k_{1}$ /moderate dissociation $t_{1 / 2}$ combinations.

Binding of the two pharmacophores of a bivalent ligand to their target sites will also result in a marked increase in its overall target residence time, which can be attributed to the propensity of a freshly dissociated pharmacophore to undergo rebinding to its cognate target site because both have to remain in close proximity as long as the other pharmacophore is still bound. The residence time of any ligand can also be increased by diffusion-related rebinding mechanisms, but not to the same extent as in the 'forced proximity' situation because dissociated molecules only briefly remain nearby their target sites. Because of the central role of rebinding events in both mechanisms, the residence time of affected radioligands can be reduced in a concentration-dependent fashion by adding competitive ligands. While this latter behaviour is unmistakably a result of competitive interactions in the present simulations, it is still considered by some to represent crucial evidence for the occurrence of allosteric interactions (Urizar et al., 2005; De Meyts et al., 2009). Hence, great care must be taken when interpreting this type of experimental observations.

In terms of utilizing this information to aid the design of more efficacious heterobivalent therapeutic agents, it appears that there is a balance to consider when choosing the correct kinetic properties of the individual pharmacophores. Depending on its pharmacokinetic half-life in the body, a heterobivalent ligand built from very high affinity, slowly equilibrating pharmacophores may not have sufficient time to achieve equilibrium with the target. Its potency could therefore be less than expected. Such a heterobivalent ligand would, however, have significant advantages in terms of residence time at the target. This combination of high affinity and slow dissociation may benefit the therapeutic efficacy of a drug because of its potentially high target selectivity and long duration of action (Copeland et al., 2006; Swinney, 2006; 2008; 2009; Zhang and Monsma, 2009; Copeland, 2012). Slow dissociating bivalent ligands that have minimal intrinsic adverse events are thus expected to be highly efficient for therapies that require continuing, elevated levels of target occupancy. 


\section{Acknowledgements}

We are most obliged to the Fonds voor Wetenschappelijk Onderzoek Vlaanderen for their kind support.

\section{Conflict of interest}

None.

\section{References}

Alexander SP, Mathie A, Peters JA (2011). Guide to Receptors and Channels (GRAC), 5th edition. Br J Pharmacol 164 (Suppl. 1): S1-S324.

Antony J, Kellershohn K, Mohr-Andrä M, Kebig A, Prilla S, Muth M et al. (2009). Dualsteric GPCR targeting: a novel route to binding and signaling pathway selectivity. FASEB J 23: 442-450.

Arranz-Plaza E, Tracy AS, Siriwardena A, Pierce JM, Boons G-J (2002). High-avidity, low-affinity multivalent interactions and the block to polyspermy in Xenopus laevis. J Am Chem Soc 124: 13035-13046.

Baker JH, Lindquist KE, Huxham LA, Kyle AH, Sy JT, Minchinton AI (2008). Direct visualization of heterogeneous extravascular distribution of trastuzumab in human epidermal growth factor receptor type 2 overexpressing xenografts. Clin Cancer Res 14: 2171-2179.

Berg HC, Purcell EM (1977). Physics of chemoreception. Biophys J 120: 193-219.

Christensen LLH (1997). Theoretical analysis of protein concentration determination using biosensor technology under conditions of partial mass transport limitation. Anal Biochem 249: $153-164$.

Christopoulos A (2002). Allosteric binding sites on cell-surface receptors: novel targets for drug discovery. Nat Rev Drug Discov 1: 198-210.

Christopoulos A, Kenakin T (2002). G protein-coupled receptor allosterism and complexing. Pharmacol Rev 54: 323-374.

Coombs D, Goldstein B (2004). Effects of geometry of the immunological synapse on the delivery of effector molecules. Biophys J 87: 2215-2220.

Copeland RA (2012). Conformational adaptation in drug-target interactions and residence time. Future Med Chem 3: 1491-1501.

Copeland RA, Pompliano DL, Meek TD (2006). Drug-target residence time and its implications for lead optimization. Nat Rev Drug Discov 5: 730-739.

De Meyts P, Roth J, Neville DM Jr, Gavin JR 3rd, Lesniak MA (1973). Insulin interactions with its receptors: experimental evidence for negative cooperativity. Biochem Biophys Res Commun 55: $154-161$.

De Meyts P, Bianco R, Roth J (1977). Site-site interactions among insulin receptors. Characterization of the negative cooperativity. J Biol Chem 251: 1877-1888.

De Meyts P, Gauguin L, Manegold Svendsen A, Sarhan M, Knudsen L, Nohr J et al. (2009). Structural basis of allosteric ligand-receptor interactions in the insulin/relaxin peptide family: implications for other receptor tyrosine kinases and G-protein-coupled receptors. Ann N Y Acad Sci 1160: 45-53.

Disingrini T, Muth M, Dallanoce C, Barocelli E, Bertoni S, Kellershohn K et al. (2006). Design, synthesis, and action of oxotremorine-related hybrid-type allosteric modulators of muscarinic acetylcholine receptors. J Med Chem 49: 366-372.

Dwir O, Solomon A, Mangan S, Kansas GS, Schwarz US, Alon R (2003). Avidity enhancement of L-selectin bonds by flow: shear-promoted rotation of leukocytes turn labile bonds into functional tethers. J Cell Biol 163: 649-659.

Fierens FLP, Vanderheyden PML, De Backer J-P, Vauquelin G (1999). Binding of the antagonist $\left[{ }^{3} \mathrm{H}\right]$ candesartan to angiotensin II $\mathrm{AT}_{1}$ receptor-transfected Chinese hamster ovary cells. Eur J Pharmacol 367: 413-422.

Frost JJ, Wagner HR Jr (1984). Kinetics of binding to opiate receptors in vivo predicted from in vitro parameters. Brain Res 305: $1-11$.

Fujimori K, Covell DC, Fletcher JE, Weinstein JN (1989). Modeling analysis of the global and microscopic distribution of immunoglobulin $\mathrm{G}, \mathrm{F}\left(\mathrm{ab}^{\prime}\right) 2$, and Fab in tumors. Cancer Res 49: 5656-5663.

Goldstein B, Dembo M (1995). Approximating the effects of diffusion on reversible reactions at the cell surface: ligand-receptor kinetics. Biophys J 68: 1222-1230.

Gregory P, Adams GP, Tai M-S, McCartney JE, Marks JD, Stafford WF III (2006). Avidity-mediated enhancement of in vivo tumor targeting by single-chain Fv dimers. Clin Cancer Res 12: $1599-1605$.

Holliger P, Hudson PJ (2005). Engineered antibody fragments and the rise of single domains. Nat Biotechnol 23: 1126-1136.

Hrabctová S, Nicholson C (2004). Contribution of dead-space microdomains to tortuosity of brain extracellular space. Neurochem Int 45: 467-477.

Jähnichen S, Blanchetot C, Maussang D, Gonzalez-Pajuelo M, Chow KY, Bosch L et al. (2010). CXCR4 nanobodies (VHH-based single variable domains) potently inhibit chemotaxis and HIV-1 replication and mobilize stem cells. Proc Natl Acad Sci U S A 107: 20565-20570.

Juweid M, Neumann R, Paik C, Perez-Bacete MJ, Sato J, van Osdol W et al. (1992). Micropharmacology of monoclonal antibodies in solid tumors: direct experimental evidence for a binding site barrier. Cancer Res 52: 5144-5153.

Kaufman EN, Jain RK (1992). Effect of bivalent interaction upon apparent antibody affinity: experimental confirmation of a theory using fluorescence photobleaching and implications for antibody binding assays. Cancer Res 52: 4157-4167.

Kenakin TP (2009). Cellular assays as portals to seven-transmembrane receptor-based drug discovery. Nat Rev Drug Discov 8: 617-626.

Kramer RH, Karpen JW (1998). Spanning biding sites on allosteric proteins with polymer-linked ligand dimers. Nature 395: 710-713.

Limbird LE, Lefkowitz RJ (1976). Negative cooperativity among $\beta$-adrenergic receptors in frog erythrocyte membrane. J Biol Chem 251: 5007-5014.

Müller KM, Arndt KM, Plückthun A (1998). Model and simulation of multivalent binding to fixed ligands. Anal Biochem 261: 149-158. 
Pack P, Müller K, Zahn R, Plückthun A (1995). Tetravalent miniantibodies with high avidity assembling in Escherichia coli. J Mol Biol 246: 28-34.

Packeu A, De Backer J-P, Van Liefde I, Vanderheyden P, Vauquelin $\mathrm{G}$ (2008). Antagonist-dopamine $\mathrm{D}_{2 \mathrm{~L}}$ - receptor interactions in intact cells. Biochem Pharmacol 75: 2192-2203.

Perry DC, Mullis KB, Øie S, Sadée W (1980). Opiate antagonist receptor binding in vivo: evidence for a new receptor binding model. Brain Res 199: 49-61.

Plückthun A, Pack P (1997). New protein engineering approaches to multivalent and bispecific antibody fragments. Immunotechnology 3: 83-105.

Rudnick SI, Adams GP (2009). Affinity and avidity in antibody-based tumor targeting. Cancer Biother Radiopharm 24: 155-161.

Schuck P, Minton AP (1996). Analysis of mass transport-limited binding kinetics in evanescent wave biosensors. Anal Biochem 240: 262-272.

Shinohara Y, Hasegawa Y, Kaku H, Shibuya N (1997). Elucidation of the mechanism enhancing the avidity of lectin with

oligosaccharides on the solid phase surface. Glycobiology 7: 1201-1208

Spivak CE, Oz M, Beglan CL, Shrager RI (2006). Diffusion delays and unstirred layer effects at monolayer cultures of Chinese hamster ovary cells: radioligand binding, confocal microscopy, and mathematical simulations. Cell Biochem Biophys 45: 43-58.

Steinfeld T, Mammen M, Smith JA, Wilson RD, Jasper JR (2007). A novel multivalent ligand that bridges the allosteric and orthosteric binding sites of the M2 muscarinic receptor. Mol Pharmacol 72: 291-302.

Steinfeld T, Hughes AD, Klein U, Smith JA, Mammen M (2011). THRX-198321 is a bifunctional muscarinic receptor antagonist and beta2-adrenoceptor agonist (MABA) that binds in a bimodal and multivalent manner. Mol Pharmacol 79: 389-399.

Swinney DC (2006). Can binding kinetics translate to a clinically differentiated drug? From theory to practice. Lett Drug Des Discov 3: 569-574.

Swinney DC (2008). Applications of binding kinetics to drug discovery: translation of binding mechanisms to clinically differentiated therapeutic responses. Int J Pharm Med 22: 23-34.

Swinney DC (2009). The role of binding kinetics in therapeutically useful drug action. Curr Opin Drug Discov Devel 12: 31-39.

Szczuka A, Packeu A, Wennerberg M, Vauquelin G (2009). Molecular mechanism of the persistent bronchodilatory effect of the partial $\beta_{2}$-adrenoceptor agonist salmeterol. Br J Pharmacol 158: 183-194.

Todorovska A, Roovers RC, Dolezal O, Kortt AA, Hoogenboom HR, Hudson PJ (2001). Design and application of diabodies, triabodies and tetrabodies for cancer targeting. J Immunol Methods 248: $47-66$.
Urizar E, Montanelli L, Loy T, Bonomi M, Swillens S, Gales C et al. (2005). Glycoprotein hormone receptors: link between receptor homodimerization and negative cooperativity. EMBO J 24: 1954-1964.

Valant C, Lane JR, Sexton PM, Christopoulos A (2012). The best of two worlds? Bitopic orthostetic/allosteric ligands of $\mathrm{G}$ protein-coupled receptors. Annu Rev Pharmacol Toxicol 52: 153-178.

Vauquelin G (2010). Rebinding: or why drugs may act longer in vivo than expected from their in vitro target residence time. Expert Opin Drug Discov 5: 927-941.

Vauquelin G, Charlton S (2010). Long-lasting target binding and rebinding as mechanisms to prolong in vivo drug action. Br J Pharmacol 161: 488-508.

Vauquelin G, Packeu A (2009). Ligands, their receptors and ... plasma membranes. Mol Cell Endocrinol 311: 1-10.

Vauquelin G, Morsing P, Fierens FLP, De Backer J-P, Vanderheyden PML (2001). A two-state receptor model for the interaction between angiotensin $\mathrm{II}_{\mathrm{AT}_{1}}$ receptors and their non-peptide antagonists. Biochem Pharmacol 61: 277-284.

Weinstein JN, Eger RR, Covell DG, Black CDV, Mulshine J, Carrasquillo JA et al. (1987). The pharmacology of monoclonal antibodies. Ann N Y Acad Sci 507: 199-210.

Wennerberg M, Cheng L, Hjorth S, Clapham JC, Ballendran A, Vauquelin $G$ (2010). Binding properties of antagonists to $\mathrm{CB}_{1}$ cannabinoid receptors in intact cells. Fundam Clin Pharmacol 25: 200-210.

Zhang J, Tanha J, Hirama T, Khieu NH, To R, Tong-Sevinc H et al. (2004). Pentamerization of single-domain antibodies from phage libraries: a novel strategy for the rapid generation of high-avidity antibody reagents. J Mol Biol 335: 49-56.

Zhang R, Monsma F (2009). The importance of drug-target residence time. Curr Opin Drug Discov Devel 12: 488-496.

Zichi D, Eaton B, Singer B, Gold L (2008). Proteomics and diagnostics: let's get specific, again. Curr Opin Chem Biol 12: 78-85.

Zuckier LS, Berkowitz EZ, Sattenberg RJ, Zhao QH, Deng HF, Scharff MD (2000). Influence of affinity and antigen density on antibody localization in a modifiable tumor targeting model. Cancer Res 60: 7008-7013.

\section{Supporting information}

Additional Supporting Information may be found in the online version of this article at the publisher's web-site:

Appendix S1 Differential equations that relate to the models shown in Schemes 1 and 2 . 